\title{
El sector no lucrativo en el marco de la nueva era tecnológica: el desafío del Crowdfunding social en España. Análisis del caso de la asociación Alba Pérez, lucha contra el cáncer infantil
}

\author{
Antonia Sajardo Moreno ${ }^{1}$, Marta Gil Ibáñez ${ }^{2}$ y Salvador Pérez Sempere ${ }^{3}$
}

Recibido: 3 de febrero de 2017 / Aceptado: 2 de mayo de 2017

\begin{abstract}
Resumen. Los albores del siglo XXI se presentan a las economías y las sociedades en el marco de un desafiante doble cambio de paradigma. Uno, alentado por el neoliberalismo gobernante en gran parte del mundo avanzado, que ha provocado que la propia sociedad admita la arresponsabilidad de las estructuras gobernantes en la prestación de los servicios que componen el Estado del bienestar. Otro, tecnológico, que ha cambiado el modo en que la sociedad se informa, se comunica y se relaciona. Frente a estas dos realidades la sociedad civil ha tomado conciencia de su papel transformador e impulsor de cambios, así como de las crecientes necesidades de atención social que han surgido en este principio de siglo, lo imprescindible de atenderlas y como no, su necesidad de financiación. Es en este ámbito en el que toma una relevancia especial la aplicación de estrategias de comunicación y marketing fundamentadas en la nueva realidad tecnológica en la búsqueda de soluciones alternativas que den viabilidad a proyectos concretos. Se analiza, en el presente artículo, el caso de la Asociación Alba Pérez de lucha contra el cáncer infantil, y la utilización que realiza de las herramientas sociales que internet proporciona, para construir y dinamizar una comunidad, los alberos, comprometida con la causa que defiende la Asociación y dispuesta a facilitar recursos para la consecución de su objetivo. En este contexto analizaremos la dinámica operativa de las herramientas del crowdfunding y de Marketing y comunicación, así como sus factores de éxito. El objetivo de este trabajo es ofrecer un ejemplo de buenas prácticas en el desarrollo de técnicas de fundraising, como es el crowdfunding, que permita al Sector No Lucrativo trazar de acuerdo a su realidad, recursos y objetivos destronando así el mito de la necesidad absoluta de financiación pública, en el cumplimiento de su misión.
\end{abstract}

Palabras clave: Sector No Lucrativo; tecnología; crowdfunding; fundraising; marketing; redes sociales.

Claves Econlit: L31; O3; G21; G29.

[en] The non-profit sector in the framework of the new technological age: the challenge of social Crowdfunding in Spain. analysis of the case of the asociation Alba Pérez, fight against childhood cancer

Abstract. The dawn of the 21st century is presented to economies and societies within the framework of a challenging double paradigm shift. One, encouraged by the ruling neoliberalism in much of the

1 Universitat de Valencia, España

Dirección de correo electrónico: antonia.sajardo@uv.es

2 Universitat de Valencia, España

3 Universitat de Valencia, España 
advanced world, has caused society itself to admit the responsibility of the governing structures in the provision of the services that make up the welfare state. Another, technological, that has changed the way society is informed, communicates and relates. Faced with these two realities, civil society has become aware of its transforming role and impelling changes, as well as the growing needs for social care that have emerged at this beginning of the century, the essential to meet them and of course, their need for funding. It is in this area that the application of communication and marketing strategies based on the new technological reality takes on a special relevance in the search for alternative solutions that give viability to concrete projects. In this article, we analyze the case of the Alba Pérez Association for the fight against childhood cancer, and its use of the social tools provided by the Internet to build and revitalize a community, the alberos, committed to the cause that Defends the Association and ready to provide resources for the achievement of its objective. In this context, we will analyze the operational dynamics of crowdfunding, marketing and communication tools, as well as their success factors. The objective of this work is to offer an example of good practices in the development of successful fundraising techniques, such as crowdfunding, which allows the Non Profit Sector to draw according to its reality, resources and objectives dethroning the myth of absolute necessity Of public financing, in the fulfillment of its mission.

Keywords: Sharing Non-Profit Sector; technology; crowdfunding; fundraising; marketing; social networks.

Sumario. 1. Introducción. 2. Crowdfunding: tecnología al servicio del fundraising en el sector no lucrativo. 3. Las herramientas de comunicación y marketing: el segundo pilar tecnológico de apoyo al sector no lucrativo. 4. Metodología del trabajo empírico. 5. Estudio de caso: la asociación Alba Pérez, lucha contra el cáncer infantil. 6. Conclusiones. 7. Referencias bibliográficas.

Cómo citar: Sajardo, A., Gil, M. y Pérez, S. (2017) El sector no lucrativo en el marco de la nueva era tecnológica: el desafío del Crowdfunding social en España. Análisis del caso de la asociación Alba Pérez, lucha contra el cáncer infantil. REVESCO. Revista de Estudios Cooperativos, $\mathrm{N}^{\circ} 124$, pp. 128151. DOI: 10.5209/REVE.56132.

\section{Introducción}

El inicio del siglo XXI se ha presentado a la sociedad como un tiempo incierto. En términos generales, este período de crisis e inseguridad ha tenido como consecuencia una situación de inestabilidad significativa al respecto de la financiación del bienestar social y la responsabilidad pública. En consecuencia, uno de los principales grupos componentes del Tercer Sector, las Entidades No Lucrativas (ENL), están experimentando una importante carga en la prestación de los servicios que han conformado, hasta día de hoy, el estado de bienestar. En este sentido, uno de los problemas más relevantes al que se enfrentan, si no el crucial porque afecta a su supervivencia, es la financiación. De forma complementaria, las entidades conviven en este difícil escenario desprovistas de toda solución financiera que posibilite su independencia de los poderes públicos dotándolas, al mismo tiempo, de poder de sostenibilidad.

Enmarcado en este contexto, surge un nuevo marco tecnológico que brinda oportunidades retadoras al problema precedente. Es el caso del uso de las nuevas tecnologías como herramienta de financiación y comunicación al servicio de las organizaciones, destacando como principal exponente el crowdfunding. Bajo este término, se reconoce al fenómeno del establecimiento de redes de personas para conseguir dinero u otros recursos y financiar un proyecto o propósito concreto, en un plazo determinado de tiempo. Pese a las posibilidades que nacen de la 
utilización de las redes para las ENLs, este ámbito apenas ha sido analizado científicamente, siendo un objeto de estudio con una proyección importante y sobre el que se hace necesario desarrollar investigaciones experimentales que se conviertan en referentes en el ámbito (Arroyo, Baladrón y Martín, 2013: 78).

En este sentido y con el fin de exprimir al máximo las posibilidades generadas por las herramientas de crowdfunding, se convierte en una estrategia fundamental la gestión adecuada de la comunicación con el fin de generar transparencia dentro y fuera de las entidades no lucrativas. Como consecuencia, estas entidades serán capaces de mejorar la imagen como percepción pública a corto plazo, aumentar la confianza a medio plazo y consolidar la reputación y estabilidad financiera a largo plazo (Herranz de la Casa, 2007). Así, este artículo parte de la premisa del aprovechamiento de las herramientas de crowdfunding ante la escasez financiera y dentro de él, la completa integración de una excelente gestión de marketing y comunicación como apoyo y recurso de éxito.

El presente trabajo propone un esquema de implementación de las herramientas de crowdfunding como respuesta a las necesidades financieras de las ENLs capaz de marcar la diferencia y asegurar la competitividad, innovación y sostenibilidad de las entidades (Fundación PwC, 2014). Las páginas que siguen se estructuran de la siguiente forma. Tras la presente introducción, el apartado segundo realiza un análisis pormenorizado de las herramientas de crowdfunding como tecnología al servicio del fundraising en el Sector No Lucrativo. A lo largo del tercer apartado se desarrolla una perspectiva general del marketing y la comunicación como herramienta de gestión y transparencia en dichas organizaciones. Una vez completada la base teórica, a continuación el cuarto apartado expone y analiza todo lo relativo al estudio de caso elaborado, que exige detallar sus fundamentos concretos, los resultados observados y las propuestas que se establecen. Por último, se recogen las principales conclusiones y futuras líneas de investigación.

\section{Crowdfunding: tecnología al servicio del fundraising en el sector no lucrativo}

El comportamiento de la sociedad actual ha sufrido una importante transformación, gracias en gran parte al nuevo paradigma tecnológico en el que se está desarrollando la vida de una parte importante de la ciudadanía en la actualidad. El flujo de transmisión de iniciativas sociales se ha visto invertido en muchos casos, dando paso a los movimientos conocidos como grassroots, iniciativas que surgen de una base social que comparte valores y objetivos concretos, en torno a proyectos específicos y que son capaces de escalar en el tradicional edificio jerárquico existente, en los esquemas de poder que se extienden en la mayor parte de las estructuras sociales actuales (Batliwala, 2002).

En el contexto de la acción social, esta nueva sociedad hiperconectada permite la creación de comunidades virtuales en torno a principios, valores y objetivos concretos. La correcta utilización de las herramientas sociales ${ }^{4}$ presentes en Internet posibilitan una amplia diseminación de los mismos en la red, y la

$4 \quad$ Facebook, twitter, entre otras. 
captación de este modo de perfiles afines a los mismos que sean susceptibles de su integración en estas pseudo comunidades.

En torno a esta realidad surge el crowdfunding como una herramienta dirigida principalmente al logro de la financiación necesaria, para la puesta en funcionamiento de proyectos determinados en un plazo temporal concreto, permitiendo a las ideas creativas, que no encajan en los patrones de la financiación tradicional, obtener dinero a través del apoyo y la solidaridad de grandes grupos de personas. En el ámbito social y de ayuda al desarrollo, integra adicionalmente la posibilidad de incremento de la base social cuantitativa (número de personas vinculadas a un proyecto) y cualitativamente (número de personas implicadas o participando de alguna forma en estos proyectos) (Cejudo y Ramil, 2013). De este modo, aporta parte de la solución a los dos obstáculos a los que, según la Plataforma del Tercer Sector, este debe encontrar solución entre 2013 y 2016 (PETS, 2012).

Bajo el término crowdfunding se identifica al fenómeno de las personas que hacen red para conseguir dinero $\mathrm{u}$ otros recursos $\mathrm{y}$ financiar un proyecto $\mathrm{o}$ propósito concreto, en un plazo determinado de tiempo. El crowdfunding permite a organizaciones e individuos conseguir recursos de una gran cantidad de pequeños aportantes comprometidos, más que de unos pocos grandes (Asociación Europea de Crowdfunding, 2013) ${ }^{5}$. Se articula bajo la idea de expandir la base de financiadores de un proyecto, en lugar de estrecharlo a una pequeña cantidad de los mismos. Según Caldbeck (2012), permite financiar un proyecto o negocio logrando una gran cantidad de pequeñas aportaciones de recursos procedentes de un gran número de individuos. De esta manera, se ubica en la base misma de la pirámide de la financiación, desdibujando su forma y convirtiéndola en un amplio rectángulo.

Si bien es un modelo de captación de recursos utilizado por la humanidad en numerosas ocasiones (Davies, 2014: 31), ha sido con la aparición de las redes sociales y la automatización de los pagos online ${ }^{6}$ cuando se ha producido el despliegue real de este modelo de obtención de recursos.

Los principios que incorpora el crowdfunding, junto con las formas de trabajo que propone, facilitan el acercamiento entre la sociedad y los proyectos sociales que apoya. Estos son principalmente (Sajardo y Pérez, 2014):

1. La creación de una comunidad alrededor de los proyectos (frente a la comunidad alrededor de la organización que implican las donaciones tradicionales).

2. La lógica del crowdsourcing, o de multitudes inteligentes colaborando en pro de un proyecto social común.

3. El ciudadano asume un nuevo papel de participación activa en los proyectos, apareciendo la figura del prosumer ${ }^{7}$.

4. Nuevos modelos de proyectos para nuevas formas de participación (voluntariado) y fundraising (financiación).

http://crowdfunding.is/.

Las herramientas más utilizadas en el crowdfunding son Paypal, Amazon Payments y Wepay

Término acuñado en 1980 por Alvin Toffler que define a los consumidores que actúan de un modo activo para

la mejora y diseño de las mercancías o servicios presentes en el mercado. PROducer+conSUMER 
5. Unión entre las tradicionales y las más novedosas formas de participación: voluntariado, implicación, donación, activismo, redes, etc.

El término crowdfunding da cobertura a una amplia diversidad de proyectos y enfoques de tratamiento de los mismos. En base a ello y dependiendo de la variable considerada, se puede realizar una ordenación taxonómica en uno u otro sentido. Atendiendo al objetivo que persigue el aportante o financiador del proyecto, se identifican cuatro modelos de crowdfunding (The Crowdfunding Industry Report, 2012).

1. Modelo crowdfunding por acciones (Equity-based crowdfunding), en cuyo contexto los aportantes de fondos reciben compensación en forma de reparto de ingresos o beneficios. El objetivo para el financiador es el mero retorno financiero, constituyendo un sistema de inversión con opción a recibir participaciones o beneficios de la comercialización del output del proyecto. Este modelo ha adquirido un enorme potencial internacional a raíz de la aprobación en Estados Unidos de la Jumpstart our Businesses Startups (JOBS) en 2012.

2. Modelo crowdfunding por préstamos (Lending-based crowdfunding), donde los aportantes de fondos reciben ingresos periódicos, y esperan recuperar la inversión inicial, con pago de intereses o no.

3. Modelo por recompensas (Reward-based crowdfunding, en el cual los colaboradores reciben recompensas no monetarias (pre ventas de una innovación, primera edición...).

4. Modelo crowdfunding por donaciones (Donation-based crowdfunding), merced al cual los aportantes poseen una motivación filantrópica, sin esperar nada tangible a cambio, solo les es importante la causa que financian.

El crowdfunding social, marco en el cual se ubica el presente trabajo, queda dentro del crowdfunding de donaciones, en el que el retorno que obtiene el donante, pieza clave para su participación en él (lanzanos.com, 2014) consiste en un agradecimiento o reconocimiento intangible. Por otro lado, al centrar la clasificación en la gestión del resultado, se identifican dos modelos de plataformas de crowdfunding.

1. El Modelo de "todo o nada" 8 , en cuyo contexto el promotor del proyecto, persona o entidad, establece un importe mínimo para la puesta en funcionamiento del proyecto, y la plataforma le dará acceso a él solamente en el caso de que se alcance el montante establecido, es decir, si el proyecto ha tenido éxito. Si el proyecto no ha alcanzado la meta financiera determinada, el dinero se devuelve a sus participantes y el creador no recibe nada (Sistema de Pledges). 
2. Modelo de "todo cuenta" ${ }^{9}$ donde el creador del proyecto recibe la suma que ha logrado recaudar en el tiempo limitado, aunque no se haya alcanzado el resultado establecido como objetivo.

En la operativa del crowdfunding social, la entidad no lucrativa confecciona la idea o proyecto a financiar, es decir, describe su objetivo sintéticamente ${ }^{10}$, indicando la cantidad necesaria para llevarlo a cabo, el tiempo de recaudación establecido y el plan de recompensas que se quiere ofrecer a cada persona que aporte dinero según la cantidad que decida donar. Todo ello se implementa en una plataforma web, proveedora de servicios de crowdfunding, que validará y hará pública la campaña correspondiente mediante la utilización de estrategias propias.

Si bien el crowdfunding, como se concibe hoy en día, depende completamente de la existencia de una infraestructura tecnológica en Internet que le de soporte, no es menos cierto que el desarrollo exitoso de cualquier campaña va a precisar de un componente de acciones offline. Por ello es imprescindible el diseño y puesta en funcionamiento de un plan de comunicación (Cejudo y Ramil, 2013) online y offline que permita escrutar los componentes reales de la comunidad sobre la que queremos actuar, ganar su apoyo e incitarles a colaborar en la difusión de la campaña, cuando no de la Entidad No Lucrativa beneficiaria de la misma. La correcta utilización de estrategias de comunicación adecuadas, va a posibilitar la implicación de los propios donantes en las acciones de comunicación y difusión, tanto de la campaña como del proyecto finalizado, sentando así las bases para la generación de una comunidad implicada en los proyectos de la propia organización (Ibídem).

Moisseyev (2013) concluye en su estudio con la existencia real de relaciones directamente proporcionales entre la actividad en los medios sociales (principalmente Facebook y Twitter) y el grado de éxito alcanzado en las campañas, gracias por una parte al impulso de difusión que aportan a la misma y, por otra, a la creación de confianza en los participantes en las campañas, en base a las opiniones y soporte existentes en las redes sociales.

Para la segura obtención del éxito en la campaña de crowdfunding, es imprescindible la correcta concepción y transmisión de la idea sobre la que se asienta el proyecto. La utilización correcta de técnicas de storytelling contribuye la adecuada difusión de la misma. Las plataformas proporcionan a los promotores de los proyectos una página de proyecto en la que incluir los vídeos, imágenes y actualizaciones sobre el proyecto que les permita difundir y mantenerlo con vida (kickstarter.com, 2014). Esta es la parte central sobre la que se asienta todo el plan de trabajo de la campaña de crowdfunding y cuenta igualmente con herramientas que facilitan la difusión de la misma en las redes sociales, incluso para la inclusión de algún widget ${ }^{11}$ del proyecto en blogs y páginas afines que deseen colaborar.

Una vez se hace pública la campaña en la plataforma de crowdfunding, el promotor de la misma, persona o entidad, asume una profusa labor de difusión del proyecto. Con este objetivo, una correcta utilización de estrategias de difusión en

\footnotetext{
Ejemplo: www.indiegogo.com

Frecuentemente se utilizan medios audiovisuales a través de la metodología del storytelling

11 Fragmento de código insertable fácilmente en los CMS (Content Management System) más utilizados en los blogs personales, por ejemplo Wordpress
} 
medios sociales facilitará, o mejor dicho, posibilitará una difusión viral de la misma. El objetivo de todo este trabajo no es otro que el de acopio de la financiación, solicitada dentro del plazo temporal establecido.

Aquellas campañas que alcanzan su objetivo finalizado el período de vigencia de la misma, pasan a una fase final en la que la entidad no lucrativa que la ha creado, o que es beneficiaria de la misma, recibe los fondos aportados por los mecenas y comienza la entrega de las recompensas, en el caso de que existan, a las que se haya comprometido con ellos. No obstante, estas recompensas, en la opción social del crowdfunding, suelen consistir en meros reconocimientos y agradecimientos intangibles. En el caso de que la campaña esté englobada dentro del modelo "todo o nada" y ésta no haya sido exitosa, es decir, que no haya alcanzado el importe objetivo que se hubiese fijado, los fondos comprometidos por los mecenas no son recaudados y la entidad beneficiaria no recibe la financiación. No obstante, una vez más, en el modelo social del crowdfunding también puede aparecer la opción "todo vale", en cuyo caso, sea cual sea el porcentaje alcanzado sobre el objetivo del proyecto, la entidad beneficiaria recibirá el importe comprometido por los mecenas.

Las propias plataformas de crowdfunding aplican un porcentaje a los importes recaudados en las campañas para su sostenimiento. Este porcentaje suele estar en torno al 5\%, dependiendo del tipo de proyecto, solidario o no, y de la plataforma en que se desarrolle. Otras plataformas, estas sí de crowdfunding social, soportan sus gastos mediante aportaciones de fundaciones, pudiendo de este modo no cargar ningún coste a los proyectos que se desarrollan en ellas. Es de resaltar el modelo de financiación de la plataforma teaming.net que, adicionalmente al apoyo de empresas privadas, cuenta con su propio team ${ }^{12}$ implicando así a los mecenas participantes en los diferentes proyectos existentes en ella para su sostenimiento.

Existen numerosas plataformas de crowdfunding con fines sociales que operan en España, bien sean nacionales o foráneas que cuentan con actividad aquí. Igualmente otras plataformas de objetivos generales dan un tratamiento especial a los proyectos con fines solidarios, especialmente con una reducción de los porcentajes que cobran a los promotores por el éxito de las campañas. Esto abre un amplio abanico de posibilidades de captación de fondos para la mayoría de las iniciativas de carácter solidario que puedan presentarse.

Como se ha constatado en los párrafos anteriores, en todo proyecto de crowdfunding aparecen tres sujetos principales implicados en él:

- $\quad$ La entidad no lucrativa que busca financiación para su proyecto ${ }^{13}$.

- $\quad$ El colectivo de personas que creen en el proyecto y lo apoyan aportando fondos (aportantes o financiadores) ${ }^{14}$, a cambio de una recompensa inmaterial (de naturaleza psicológica), o tangible (no económica-descargas, copia libro o audiovisual, entradas al evento, camiseta, figurar en los créditos...- o económica -retorno financiero: intereses, rentas, recuperar importe inversión).

\footnotetext{
Conjunto de donantes que apoyan un proyecto mediante la aportación recurrente de $1 €$ al mes

Promotor del proyecto

En el crowdfunding social nos referiremos a ellos como mecenas
} 
- La plataforma tecnológica que facilita el contacto entre unos y otros, llevando a cabo las tareas de selección de proyectos, asesoramiento, licencia de software u otras, por las que cobra comisión, oscilando generalmente entre el $5 \%$ y $9 \%$.

La mecánica operativa del crowdfunding es muy sencilla, pero conlleva una gran dedicación de recursos durante el período de vida de la campaña para conseguir alcanzar el objetivo marcado (startsomegood.com, 2014). Esto ha hecho que, en los casos de crowdfunding social como algunos en los que aparece la Asociación Alba Pérez como beneficiario, aparezca un cuarto sujeto implicado en la promoción de un proyecto. Se trata de personas ajenas a la Entidad No Lucrativa pero que deciden lanzar y gestionar campañas que tienen como beneficiario dicha entidad. Esta figura está muy presente en plataformas como migranodearena.org o teamning.net. Estos proyectos de crowdfunding promovidos por donantes, suelen partir de comunidades de donantes creadas en campañas anteriores, el community fundraising, que poseen capacidad para emprender por su cuenta y riesgo, nuevas campañas de crowdfunding (Cejudo y Ramil, 2013).

A la hora de plantear el crowdfunding como un medio para la sostenibilidad de una entidad no lucrativa y de los proyectos que esta promueve, es imprescindible tener en cuenta la escala de los proyectos financiados por este medio, así como la recurrencia de participación en los mismos por parte de los mecenas. Esto va a depender en gran parte de la plataforma proveedora de servicios de crowdfunding en la que se vaya a desarrollar la campaña. En plataformas exclusivas de crowdfunding social en España, como son migranodearena.org y microdonaciones.hazloposible.org, aparecen escalas medias inferiores a los $4.000 €$ (Sajardo y Pérez, 2014), otras como miaportacion.org se basan en microproyectos de valores inferiores a los $500 €$.

El crowdfunding es un fenómeno neonato, lo que da pie a la introducción de innovaciones en su aplicación y enfoque, también dentro del ámbito de los objetivos sociales. Esto lo ha convertido en una realidad con un dinamismo sorprendente y que, a nivel de desarrollo y expansión, está alcanzando cotas muy elevadas. De hecho, el crowdfunding conjunto, englobando todo tipo de iniciativas y considerando un ámbito geográfico global, ha experimentado un crecimiento del $101,47 \%$ en fondos comprometidos y un $49,75 \%$ en recaudados en el primer trimestre del año 2014. El período de tiempo que precisa para duplicar su volumen es de 60 días (The crowdatacenter, 2014).

Como fenómeno de reciente aparición, la bibliografía y el respaldo empírico a la misma existente con referencia al crowdfunding es más bien limitada, más aún en el ámbito de la utilización del mismo para fines sociales, como es el objetivo del presente artículo. Por este motivo, se ha seleccionado esta asociación para analizar el motivo de su viabilidad como herramienta complementaria para la financiación de las Entidades No Lucrativas. 


\section{Las herramientas de comunicación y marketing: el segundo pilar tecnológico de apoyo al sector no lucrativo}

La gestión de la comunicación como una estrategia dentro de las ENLs es un elemento cada día más necesario y relevante dentro del sector no lucrativo (Herranz de la Casa, 2007). En este sentido, la comunicación se enmarca como una de las variables de una disciplina de mayor tamaño, el marketing. A lo largo del tiempo, la definición de marketing ha experimentado importantes cambios, una evolución visible que se torna más comprensible al analizar como la definición del concepto ha ido variando con el tiempo. Para ello, se analizan las definiciones expuestas en las últimas décadas por la American Marketing Association (AMA):

- 1969: el marketing es el resultado de las actividades comerciales que dirigen el flujo de bienes y servicios desde el productor hasta el consumidor o usuario.

- $\quad$ 1985: el marketing es el proceso de planificación y ejecución de todo lo relativo al concepto, precio, promoción y distribución de ideas, bienes o servicios con el fin de crear intercambios y cumplir los objetivos.

2004: el marketing es una función organizativa y un conjunto de procesos para crear, comunicar, dar valor a los consumidores y gestionar las relaciones con ellos de forma que beneficie a la organización y a sus colaboradores.

2013: el marketing es la actividad, conjunto de instituciones y procesos para crear, comunicar, deliberar e intercambiar recursos con un valor a largo plazo para los consumidores, clientes, colaboradores y la sociedad en general.

En relación con las diferentes tipologías de marketing existentes, se diferencian diversos tipos (Vázquez, 2004). En primer lugar, tomando en cuenta el agente principal de intercambio, destacan tres tipos de marketing:

Figura. 1. Tipología de marketing en función del agente principal de intercambio.

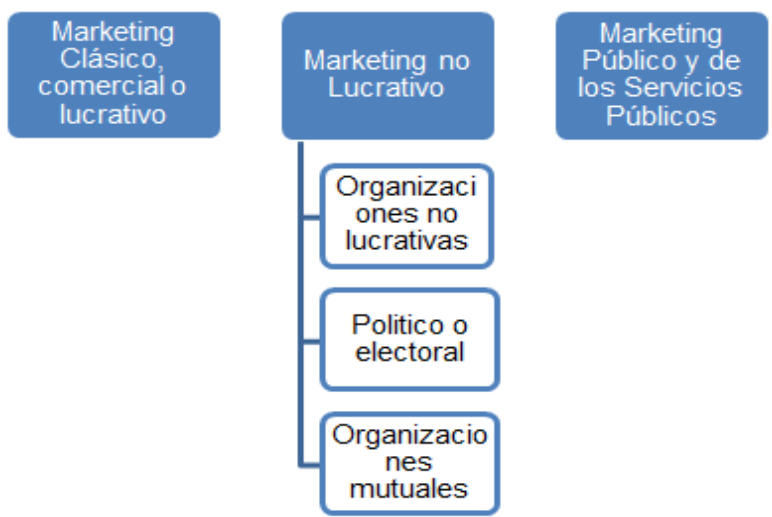

Fuente: Vázquez (2004) 
Este trabajo considera únicamente el segundo grupo ya que es el único que toma en cuenta el Sector No Lucrativo. Para continuar y como segunda clasificación, se toma en cuenta el objeto de intercambio, diferenciando esta vez dos tipos de marketing:

Figura. 2. Tipología de Marketing en función del objeto de intercambio.

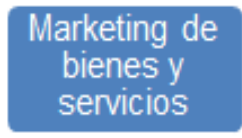

Fuente: Vázquez (2004)

\section{Marketing \\ social}

El marketing de bienes y servicios es aquel en el cual es posible hacer una contribución y colaborar. Por otro lado, el marketing social trabaja por crear cambios o adoptar comportamientos asociativos en el público objetivo (Herranz de la Casa, 2007). El presente trabajo tiene en cuenta los dos tipos, ya que ambos están encuadrados dentro del marco del Sector No Lucrativo. En definitiva, se puede concluir que esta investigación se centra en tres tipos específicos de marketing: el no lucrativo, el de bienes y servicios y el social.

La aplicación de las técnicas de marketing es el mejor camino para las organizaciones de conseguir unos niveles de satisfacción más elevados de sus usuarios, fácilmente traducible como garantía de la existencia de presupuesto (Kotler Armstrong, Saunders y Wong, 1999). No obstante, las organizaciones únicamente recurren a su uso si están más preocupadas por las necesidades de sus clientes que por las funciones que realizan (Santesmases, 2004). Está probado que existe una relación directa entre la inversión realizada en gestionar la comunicación y la efectividad en la consecución de los objetivos de algunos programas (Edgar, 2001). No obstante y tal y como se observa en el caso de estudio de referencia, sirve de muy poco invertir en costosas campañas de comunicación, si la imagen a crear, reforzar o modificar no se confirma por la práctica en el día a día (García y Ramírez, 2001). En un futuro próximo, la supervivencia y la garantía de calidad de muchas organizaciones no lucrativas serán directamente proporcionales al uso de la transparencia como un valor en su comportamiento diario. Además, dependerá también de la forma en la que fomenten y comuniquen dicho valor a todo el conjunto de públicos de dentro y fuera de la organización (Herranz de la Casa, 2007). Con la evolución de las nuevas tecnologías y la consecuente aparición de herramientas y formas de participación como el crowdfunding, estas afirmaciones se han convertido en la esencia de las organizaciones que como respuesta a la situación financiera recesiva buscan nuevos colaboradores y fuentes de financiación.

Actualmente, el Marketing Mix se entiende como una de las ideas predominantes en las corrientes de marketing moderno, entendido como la composición del grupo de herramientas tácticas controlables que una empresa u organización maneja para producir en su público objetivo la respuesta que desea (Kotler et al., 1999). Como aparece en la siguiente ilustración, el Marketing Mix se 
divide en cuatro grupos de variables conocido debido a su nombre en inglés como las “4 p's": producto, precio, promoción y distribución.

Figura. 3. Las cuatro P's componentes del Marketing Mix.

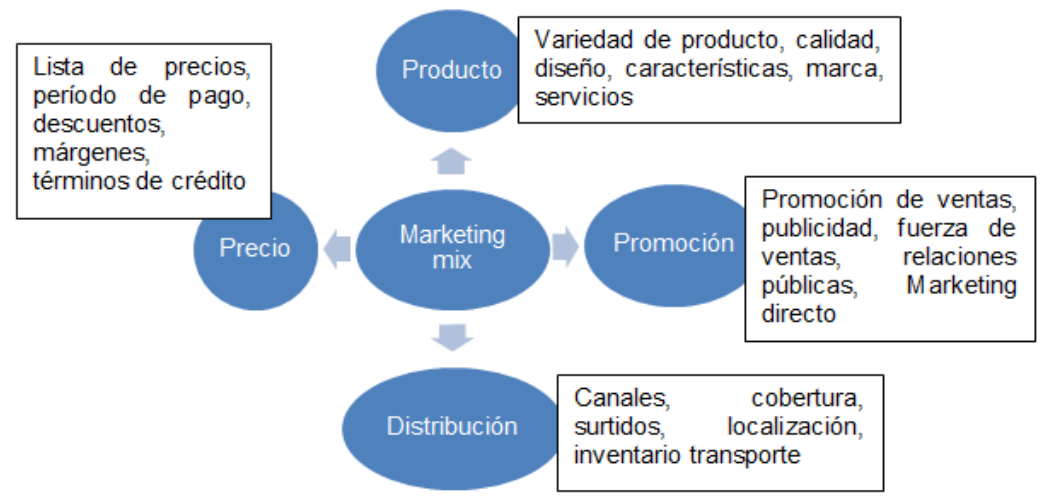

Fuente: Kotler y Keller (2006)

Las cuatro P's representan el punto de vista de las herramientas de marketing disponibles de los vendedores para influir en los compradores (Kotler y Keller, 2006). Al fin y al cabo, en el Marketing Mix se enmarca todo aquello que una organización puede hacer para influenciar en la demanda de su producto (Kotler et al., 1999). Actualmente, es el mismo tanto si se refiere a una visión lucrativa como no lucrativa. No obstante, existen diferentes aspectos que sí que presentan y hacen una diferencia entre el Marketing Mix utilizado en ambos casos. De esta forma, en el caso de entidades no lucrativas se puede especificar de la siguiente forma:

- Producto: mayoritariamente, el producto es el programa y servicio ofertado. Las organizaciones no lucrativas no son conducidas en función de su producto, si no de su misión. El reconocimiento de marca es esencial en este sector y permite mejorar sustancialmente la comerciabilidad de la entidad (Wolf, 1999). Las organizaciones no lucrativas tienen una mentalidad de Marketing "centrada en la organización” y pueden cometer un error al considerar que su producto o servicio es necesario para el mercado (Andreasen y Kotler, 2003).

- Precio: ayuda a establecer la percepción del valor de un producto o servicio. Actualmente, el precio supone lo que cuesta participar (Wolf, 1999).

- Distribución: es la localización en la cual el producto, servicio o programa se pone a disposición de los consumidores. Muchas organizaciones han de comprender y trabajar para entender que si los consumidores, clientes o integrantes no pueden o no quieren acudir a la localización predispuesta por ellos, es la propia entidad quien ha de poner el producto o servicio en un lugar accesible (Wolf, 1999). 
Promoción: es la promoción del producto, programa o servicio (Wolf, 1999). Normalmente, las organizaciones no lucrativas pueden promover su imagen, productos y programas a través de diferentes opciones: campañas mediáticas, conferencias, etc. Comúnmente, estas organizaciones no designan muchos recursos en este sentido y se sirven de herramientas de comunicación gratuita. De la abundancia de organizaciones y la dificultad existente para aparecer en otros medios ha surgido la conveniencia de buscar otros canales, cobrando relevancia primero la comunicación web (Martín, 2010) y poco después las redes sociales por el abaratamiento que supone para los procesos comunicativos de las ONG (Sampedro, 2006). En este sentido, la comunicación social es el instrumento que las ENLs cada vez más utilizan para dar a conocer sus principios y acciones y resulta clave analizar cómo las organizaciones adecuan sus usos y presupuestos de comunicación a la nueva realidad (Arroyo et al., 2013).

En el Sector No Lucrativo, cuando la implementación de las estrategias de marketing son exitosas permiten a la organización trabajar sobre su misión, conseguir los objetivos propuestos y obtener estabilidad financiera a largo plazo (Wolf, 1999). De este modo, cabe destacar la posibilidad que brinda la transversalidad de la gestión de la comunicación de interrelacionar todas las áreas de gestión de la actividad de la organización: área financiera, área de organización de actividades, área de planificación estratégica, área de recursos humanos, etc. (Herranz de la Casa, 2007). Un gran número de organizaciones no lucrativas todavía niegan la existencia de competencia alegando que esta es sólo característica del sector privado, pensando que organizaciones con actividades similares simplemente son colaboradores en la provisión de servicios sociales, no competidores. De hecho, desarrollar una adecuada estrategia de Marketing Mix para los productos y servicios es tan importante en el Sector No Lucrativo como lo es entre las empresas lucrativas. Aunque algunos investigadores comienzan a coincidir que la adopción de una perspectiva orientada de mercado es también importante para las organizaciones no lucrativas (Andreasen y Kotler, 2003), se debe argumentar que la orientación de mercado no es tan relevante como parece ya que tal y como se ha visto, su misión (producto) se define con anterioridad y no puede cambiarse en función de las necesidades del mercado.

La sociedad y todos sus sectores evolucionan de manera constante y es por ello que la adaptación a los cambios por parte de todos los agentes resulta imprescindible. Además, la velocidad experimentada es cada vez mayor, disminuyendo proporcionalmente el tiempo de respuesta disponible. En este aspecto, la gestión de las entidades no lucrativas y la implementación de las herramientas de marketing no son una excepción en absoluto. En los últimos años y consecuencia de la recesión financiera experimentada, las entidades no lucrativas han reinventado la forma de involucrar a la sociedad y establecer nuevas formas de financiación. Uno de los resultados más notables y como ya se ha visto, ha sido la utilización de Internet para la creación y puesta en marcha de plataformas de crowdfunding, herramienta dirigida a la consecución de financiación de proyectos. En este sentido y al compás de la evolución de las posibilidades de financiación y el aumento de la competencia en el sector no lucrativo, las obligaciones de las 
entidades contraídas con sus colaboradores también han aumentado. Así, el marketing en su vertiente comunicativa se establece como una herramienta esencial para trasladar de forma transparente el proyecto de la organización y poder seguir contando con el apoyo y confianza de sus socios. Es de esta forma como el seguimiento de una buena estrategia de marketing se erige como la única herramienta capaz de cerrar el círculo creado por el crowdfunding y dotarle de permanencia en el tiempo. A modo de conclusión y al igual que está ocurriendo en el mundo empresarial, se acrecienta cada vez más la obligación de trabajar sobre la comunicación y transparencia de las organizaciones para obtener el máximo rendimiento de los distintos grupos de interés, ampliando así las posibilidades para las organizaciones de obtener una estabilidad financiera (Herranz de la Casa, 2007).

\section{Metodología del trabajo empírico}

La multitud de variables que influyen en el desarrollo exitoso de una estrategia de comunicación en torno a un proyecto de índole social concreto, y su materialización a través del fenómeno del crowdfunding, ha llevado a optar por la metodología de análisis cualitativa. De esta manera y para poder dar respuesta a las cuestiones planteadas, se recurre a la metodología de casos establecida por Yin (1989) y Rialp (2003).

La elección de esta metodología se fundamenta en la posibilidad de analizar el fenómeno objeto de estudio en su contexto real, utilizando múltiples fuentes de evidencia, cuantitativas y/o cualitativas simultáneamente (Yin, 1989; Villarreal y Landeta, 2010; Rialp, 2003). En este contexto, Chetty (1996) afirma que el método de estudio de caso es una metodología rigurosa, ya que permite analizar los fenómenos desde múltiples perspectivas y no desde la influencia de una sola variable, a la par que facilita su exploración de forma más profunda, alcanzando un conocimiento más amplio y global de cada objeto de estudio.

Se ha seleccionado el caso concreto de la Asociación Alba Pérez, lucha contra el cáncer infantil por tratarse de una ENL de reciente creación, que no cuenta con ningún apoyo de las Administraciones Públicas y que basa su estrategia de captación de recursos en una comunicación vía medios sociales, principalmente Facebook, y en la utilización, mayoritariamente, del fenómeno del crowdfunding. La asociación ha alcanzado una relevancia mediática y nivel de éxito más que suficientes como para justificar su utilización como objeto de estudio único y dar validez a las conclusiones obtenidas de un modo extensivo. A continuación, la Tabla 1 muestra la ficha técnica del estudio:

Tabla. 1. Ficha técnica del estudio

\begin{tabular}{|c|c|}
\hline $\begin{array}{c}\text { Metodología de } \\
\text { investigación }\end{array}$ & $\begin{array}{c}\text { Estudio de la Asociación Alba Pérez, lucha contra el } \\
\text { cáncer infantil. Estudio exploratorio, ilustrativo y } \\
\text { potencialmente explicativo. }\end{array}$ \\
\hline Método de recogida de & Entrevista personal con el presidente de la \\
\hline
\end{tabular}




\begin{tabular}{|c|c|}
\hline la información & $\begin{array}{l}\text { Asociación. } \\
\text { Rastreo, extracción de datos y análisis directo de los } \\
\text { proyectos en migranodearena.org, teaming.net } \\
\text { Extracción directa de la información sobre la } \\
\text { actividad de la Asociación en Facebook y Twitter. }\end{array}$ \\
\hline Informantes clave & Rafael Pérez, Presidente de la Asociación \\
\hline $\begin{array}{l}\text { Métodos de análisis de } \\
\text { la evidencia }\end{array}$ & $\begin{array}{c}\text { Básicamente de tipo cualitativo: } \\
\text { - Identificación y clasificación estructural de } \\
\text { dimensiones clave. } \\
\text { - Búsqueda de factores explicativos clave. } \\
\text { - Búsqueda de patrones de comportamiento } \\
\text { comunes. } \\
\text { - Creación de explicación teórica. } \\
\text { - Identificación de relaciones causales. } \\
\text { - Análisis de decisiones críticas. }\end{array}$ \\
\hline Enfoque científico & $\begin{array}{l}\text { Procesos deductivos, en tanto en cuanto se parte de } \\
\text { las proposiciones teóricas procedentes de la revisión } \\
\text { de las teorías. }\end{array}$ \\
\hline $\begin{array}{l}\text { Evaluación de la } \\
\text { calidad y el rigor } \\
\text { metodológico }\end{array}$ & $\begin{array}{l}\text { Validez (constructiva, interna y externa), fiabilidad, } \\
\text { consistencia (contextual y teórico-interpretativa). }\end{array}$ \\
\hline Fecha de realización & Julio - agosto 2014 \\
\hline
\end{tabular}

Fuente: adaptado de Yin (1989), Rialp (2003) y Villareal y Landeta (2010).

\section{Estudio de caso: la asociación Alba Pérez, lucha contra el cáncer infantil ${ }^{15}$}

\subsection{Circundando la sinopsis de la entidad Asociación Alba Pérez}

La Asociación Alba Pérez, lucha contra el cáncer infantil se constituye a inicios del año 2012 con el objetivo de obtener fondos que posteriormente serán donados para la investigación del cáncer infantil, especialmente la variante Sarcoma de Ewing, el segundo tipo de cáncer de huesos más común que afecta a niños y jóvenes. Actualmente, con un diagnóstico temprano y si no presenta metástasis, puede ser curado en el $80 \%$ de los casos. Sin embargo, entre el $25 \%$ y el $30 \%$ de los casos son detectados cuando ya presentan metástasis, lo que hace que el porcentaje de supervivencia descienda hasta el $30 \%$.

A Alba Pérez, nacida en julio de 2008, se le diagnosticó esta enfermedad cuando contaba con cinco meses de vida. Las dificultades encontradas por sus familiares para mantener sus puestos de trabajo, atender adecuadamente a su hija y cubrir los costes que esto les generaba, les incitó a impulsar una proposición no de ley que reconociera a las familias en esta situación especial y que concluyó con la publicación del Real Decreto 1148/2011, de 29 de julio, para la aplicación y

15 En el momento de la redacción del presente artículo la Asociación Alba Pérez se encontraba en fase de cambio de personalidad jurídica desde Asociación a Fundación. Este cambio se produjo en el año 2015 
desarrollo, en el sistema de la Seguridad Social, de la prestación económica por cuidado de menores afectados por cáncer u otra enfermedad grave. Según la Federación Española de Padres de Niños con Cáncer ${ }^{16}$, cada año se diagnostican en torno a 1.400 casos nuevos de cáncer, en todas sus variantes, en españoles de hasta 18 años.

Alcanzado este objetivo y motivados por la positiva acogida que su caso tuvo en medios de comunicación, en su entorno local y en los medios sociales ${ }^{17}$, se constituyó la Asociación con el fin de crear la beca Alba Pérez. Esta beca tiene un importe de $200.000 €$ y va dirigida a Hospitales e Institutos de Investigación que trabajen sobre esta modalidad de cáncer infantil, el Sarcoma de Ewing.

El pasado 2 de octubre de 2013 se hizo entrega de la primera beca de $200.000 €$ al equipo investigador dirigido por el Dr. Óscar Martínez Tirado del Institut d'Investigació Biomèdica de Bellvitge (IDIBELL) ${ }^{18}$. Su importe cubre los gastos de investigación de esta enfermedad durante tres años, si bien el período esperado para la obtención de resultados esperanzadores es de cinco a siete años. Por este motivo, la Asociación Alba Pérez, lucha contra el cáncer infantil se encuentra ahora mismo recogiendo fondos para una segunda beca que tendrá el mismo importe.

El número de asociados existentes en esta asociación se limita a los familiares más cercanos de la niña Alba Pérez, fallecida el 1 de agosto de 2013 a causa del cáncer que padecía. Pese a ello, Rafael Pérez, padre de la niña y presidente de la asociación, mantiene su compromiso de lucha contra ella.

A finales del año 2015, la entidad se convirtió en Fundación persiguiendo dos objetivos principales; el primero es la consecución de una mejor imagen frente a otras entidades y organismos públicos, y el segundo, facilitar a las personas y empresas que realizan donaciones, una desgravación de las mismas en sus tributos.

\subsection{Estructura del fundraising en la entidad asociativa Asociación Alba Pérez: sobre los perfiles de la herramienta del crowdfunding}

Las herramientas utilizadas por la Asociación Alba Pérez para obtener los fondos que han sido aplicados a la primera beca de $200.000 €$, ya transferida al Instituto Idibell, y la siguiente, todavía en fase de recepción de aportaciones, se basan en microdonaciones de un gran número de personas, que no tienen ninguna recompensa tangible. Estas son las características que definen el crowdfunding de donación.

Las plataformas utilizadas para ello han sido migranodearena.org y teaming.net. Paralelamente, cuenta con otro tipo de colaboraciones más tradicionales en base a donaciones puntuales, tanto de personas físicas como de empresas, proyectos de merchandising impulsados por actores externos a la Asociación Alba Pérez, eventos y sorteos. Entre este conjunto de iniciativas paralelas, que quedan fuera del objeto de estudio de este artículo, si se quiere resaltar por su naturaleza el juego Queca Viste, disponible en la plataforma Play Store de Google, cuyos autores han

\footnotetext{
http://cancerinfantil.org/ principalmente Facebook

http://www.idibell.cat/modul/noticies/en/611/the-alba-perez-association-funds-a-project-of-the-idibell-toinvestigate-ewings-sarcoma
} 
comprometido con la asociación donaciones periódicas basadas en los fondos recaudados por el mismo.

migranodearena.org es una plataforma de crowdfunding solidario (donaciones en grupo) pionera en España, que tiene como objetivo recaudar fondos a favor de diferentes ONG. Aproxima la solidaridad a los ciudadanos, facilitando una nueva forma de aportar en favor de una causa social. Estos pueden promover y liderar retos cuyo beneficiario final será la ONG seleccionada. Se articula a través del sistema de donaciones (Plataforma de Donaciones-Rewards-crowdfunding) y el método empleado es el de todo cuenta. Sus proyectos son, por lo general, de reducido nivel de necesidad financiera, oscilando de media entre los $1.000 €$ y los $3.000 €$. Esta plataforma ha sido creada por la Fundación Real Dreams ${ }^{19}$, que tiene como misión conseguir recursos económicos, de tiempo y de producto, para ayudar a las ENLs. Actúa como dinamizador de la acción social entre empresas, personas y ENLs, para contribuir a la mejora de la calidad de vida de los colectivos más desfavorecidos.

La Asociación Alba Pérez cuenta en esta plataforma con trece proyectos; tres de ellos han sido promovidos por la propia asociación con una recaudación total, en la fecha de elaboración del presente artículo, de 140.576,60 €, los otros 10 han sido promovidos por personas externas a la Asociación con una recaudación total de $10.249,92 €$.

Tabla. 2. Proyectos presentes en migranodearena.org con beneficiario Asociación Alba Pérez

\begin{tabular}{|cccccc|}
\hline Recaudado & Objetivo & Donativos & Inicio & Promotor & Estado \\
$18.933 €$ & $200.000 €$ & 519 & 16.10 .2012 & Asociación & Cerrado \\
$0 €$ & $2.000 €$ & 0 & 06.11 .2012 & Externo & Cerrado \\
$67.387 €$ & $50.000 €$ & 1.428 & 25.01 .2013 & Asociación & Cerrado \\
$0 €$ & $12 €$ & 0 & 06.04 .2013 & Externo & Cerrado \\
$0 €$ & $3.000 €$ & 0 & 12.06 .2013 & Externo & Cerrado \\
$870 €$ & $6.000 €$ & 24 & 02.07 .2013 & Externo & Cerrado \\
$54.257 €$ & $50.000 €$ & 2.049 & 15.07 .2013 & Asociación & Abierto \\
$7.325 €$ & $10.000 €$ & 156 & 25.08 .2013 & Externo & Cerrado \\
$520 €$ & $2.000 €$ & 25 & 05.03 .2014 & Externo & Cerrado \\
$5 €$ & $2.000 €$ & 1 & 22.04 .2014 & Externo & Cerrado \\
$598 €$ & $1.500 €$ & 41 & 27.05 .2014 & Externo & Cerrado \\
$20 €$ & $1.000 €$ & 3 & 28.05 .2014 & Externo & Cerrado \\
$912 €$ & $1.600 €$ & 32 & 06.06 .2014 & Externo & Abierto \\
\hline
\end{tabular}

Fuente: elaboración propia 
Figura. 4. Evolución por semanas de las donaciones en migranodearena.org a la Asociación Alba Pérez.

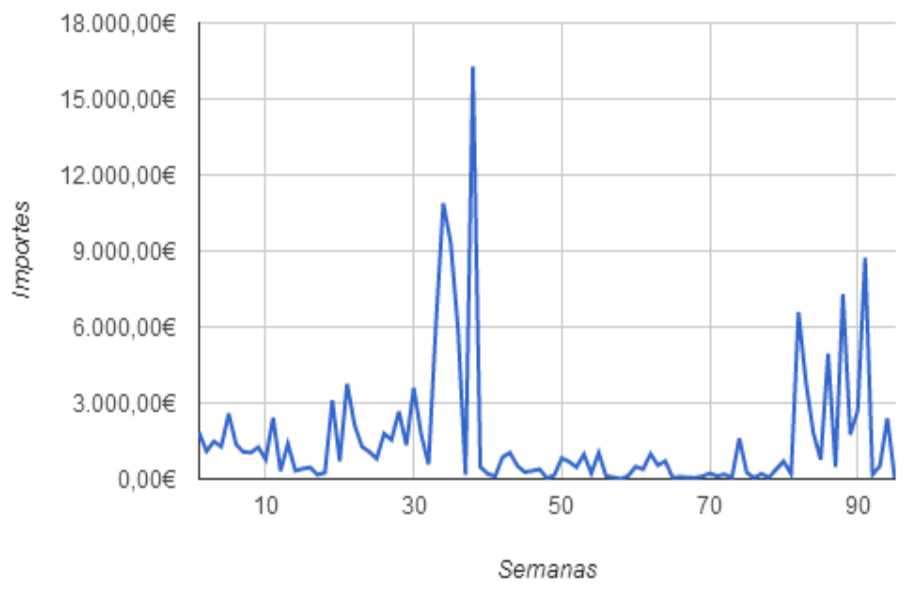

Fuente: elaboración propia

Teaming.net es una iniciativa solidaria nacida en 1988 y que permite la creación de teams, agrupaciones de donantes en torno a un proyecto concreto, cuyos integrantes donan $1 €$ al mes para dicho proyecto. La actividad de teaming comenzó en las empresas, donde sus empleados donaban de su nómina $1 €$ al mes para proyectos sociales. La aparición de teaming online, teaming.net, ha extendido esta iniciativa a cualquier persona, tanto para la promoción de proyectos de índole social como para su participación en grupos (teams) creados por terceros.

La operativa de teaming.net se basa en la figura del teaming manager, que es el creador del grupo (team), responsable de su dinamización y gestor del mismo. Esta es la figura que selecciona el proyecto a sostener y gestiona las transferencias del importe recaudado al mismo. Las aportaciones son recurrentes, mensuales.

La Asociación Alba Pérez cuenta con dos teams de apoyo en esta plataforma.

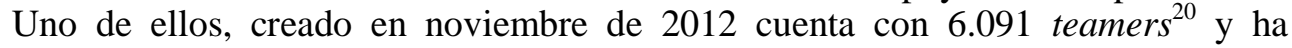
recaudado hasta la fecha $94.600 €$. El otro, creado en junio de 2013, cuenta con 1.286 teamers y ha recaudado hasta la fecha $16.434 €^{21}$. Globalmente se han recaudado $114.034 €$, cifra que se incrementa mensualmente en $7.361 €$, esto hace que en períodos de 28 meses aproximadamente, sin ningún otro ingreso, se pueda conseguir el montante de $200.000 €$, coste de la investigación a la que van dirigidos los fondos, durante 36 meses. La creación del segundo Team se produce por la limitación en las donaciones aplicadas por la plataforma y a modo de salida hacia delante para personas interesadas en aumentar su donación mensual.

Aprovechando igualmente el auge de la banca electrónica y la cada vez mayor utilización de los servicios online ofrecidos por la banca tradicional (Suárez, 2013: 17), la Asociación Alba Pérez cuenta con una página personalizada en el portal de

\footnotetext{
20 Donantes

21 A fecha de agosto de 2014
} 
una entidad bancaria española, "la Caixa", a través de la cual pueden recibir donaciones puntuales.

Figura. 5. Página web de "la Caixa" para realizar donativos puntuales a la Asociación Alba Pérez.

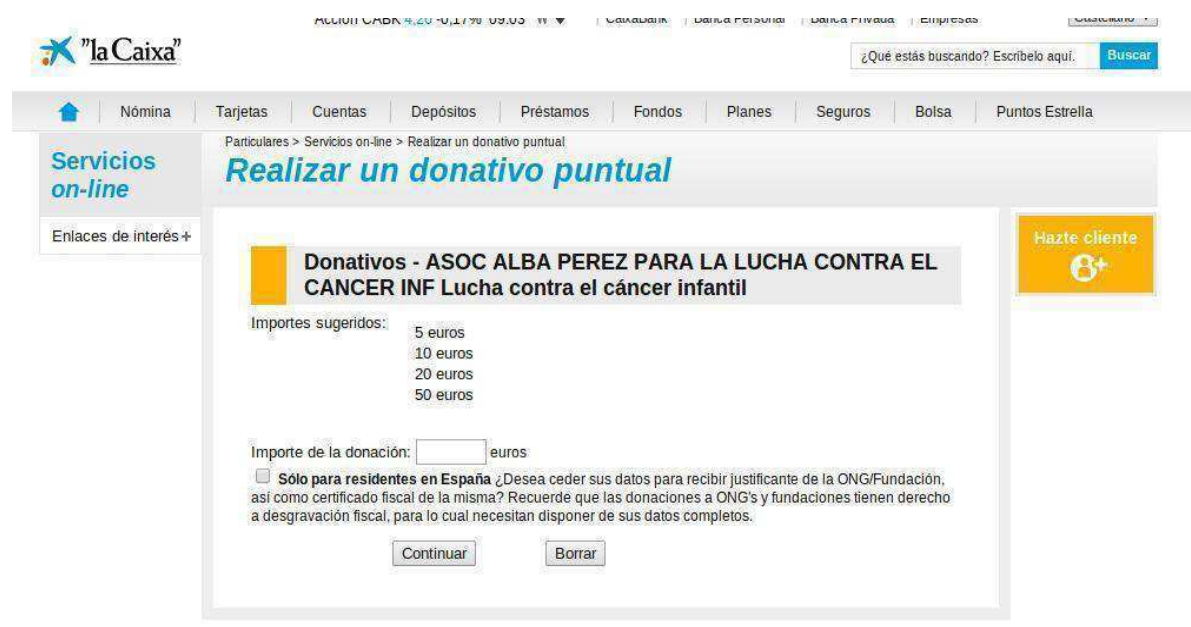

Fuente: Página web "la Caixa"22

En el mismo sentido, siendo conscientes de la importancia de los teléfonos inteligentes en el estilo de vida de la sociedad actual (Suárez, 2012) y siendo paypal uno de los sistemas de pago a través de estos dispositivos más utilizado (Suárez 2012: 57), la Asociación cuenta con su aplicación en esta plataforma. 
Figura. 6. Página web de Paypal para realizar donativos puntuales a la Asociación Alba Pérez.

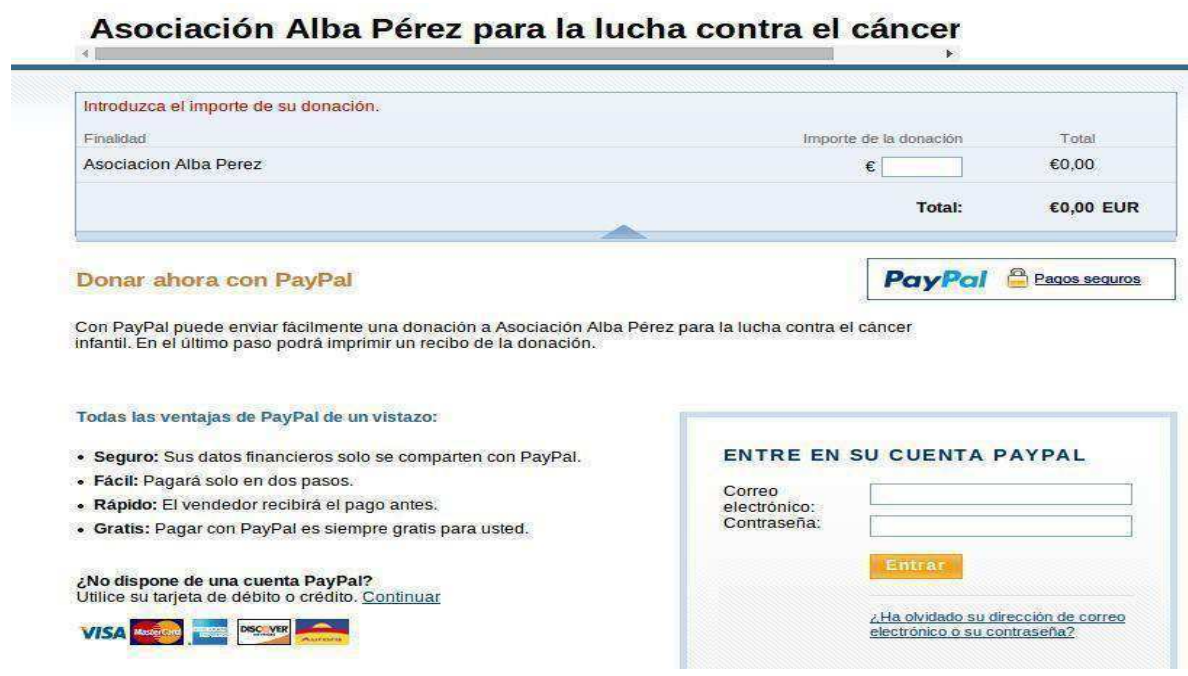

Fuente: Página web Paypal

\subsection{En relación al Marketing y comunicación en la Asociación Alba Pérez}

Como se ha analizado en el apartado anterior, esta investigación utiliza las herramientas del Marketing Mix para estudiar si la entidad de referencia implementa o no una visión de marketing con el fin de analizar su impacto en la utilización de las plataformas de crowdfunding. De esta forma, el análisis se divide en cuatro partes: producto, precio, lugar y distribución.

\section{A. El Output Ofertado}

Tal como se ha mostrado en los apartados anteriores, el producto en el caso de organizaciones no lucrativas se compone del programa o servicios ofertados. En este estudio, la asociación tiene bien definido su producto y es capaz de definir su actividad principal en una frase. No obstante, no cuentan con una misión como tal concreta y definida. En su página web, la Asociación Alba Pérez define su actividad como:

"El objetivo principal de nuestra entidad es la organización, realización y gestión de actos de promoción, divulgación, captación de fondos mediante donaciones de personas, Organismos Públicos y empresas con el fin de dedicar dichos fondos a la investigación de los cánceres infantiles, en especial el Sarcoma de Ewing, entregándolos a todos aquellos hospitales o laboratorios que se dediquen a la investigación para lograr la curación de los cánceres infantiles."

\section{B. EI Factor precio}

El factor precio establece en cierta manera la percepción del valor del producto o servicio. Se refiere tanto al importe que supone la realización del proyecto como 
al precio de lo que cuesta participar. Además, resulta imprescindible señalar en referencia al coste total del proyecto si existe un presupuesto destinado a las actividades de Marketing. En este sentido, la asociación no dispone de un presupuesto general aprobado con anterioridad, sino que su actividad principal se focaliza en recaudar el máximo dinero posible para poder ampliar en tiempo y calidad la beca de colaboración que entregan y conseguir así su objetivo principal. En consecuencia, no existe ninguna partida presupuestaria destinada exclusivamente a la realización de actividades de Marketing. Respecto al valor de lo que cuesta participar, la asociación es un claro ejemplo de la modificación en las conductas en cuanto a participación social se refiere. Wolf (1999) señalaba que un consumidor que pretenda diferenciar entre dos organizaciones del mismo campo puede a menudo llegar a concluir que aquella que dispone de precios más elevados ofrece un mejor producto o servicio. En este caso y derivado de las técnicas de innovación planteadas por el crowdfunding, sucede completamente lo contrario. La asociación busca una participación masiva en el proyecto con aportaciones pequeñas ( $1 €$ al mes). Por tanto, se concluye que el precio derivado de participar en la asociación es de carácter bajo.

\section{Las variables de distribución}

La distribución implica la localización donde el producto o servicio se hace disponible para el cliente. En este caso, la asociación lleva a cabo su proyecto de forma directa e incita a la participación de todo el mundo a través de Internet. A día de hoy, la entidad no dispone de un lugar físico, pero sí de una gran red de herramientas online.

\section{Los aspectos de promoción}

Una de los componentes más importantes en la promoción es la comunicación, dirigida principalmente a los dos grandes grupos de una entidad: interna (empleados) y externa (colaboradores y beneficiarios). La Asociación Alba Pérez no dispone de empleados, por lo que focaliza todos sus esfuerzos y recursos en la comunicación externa, principalmente hacia los colaboradores. Como principales medios de comunicación, la organización utiliza su página web y tiene una actividad muy dinámica en las redes sociales.

Otro de los componentes que destacan en la promoción es el uso de la imagen corporativa. En este caso, es la propia imagen de la niña Alba el estandarte de todas las actividades que la entidad lleva a cabo, dejando a un lado logos, tipografías y colores. Esta estrategia sigue el hilo de la actitud tomada por la organización, muy personalista y haciendo hincapié en ser completamente transparente.

Por último, la entidad trata de utilizar anuncios y publicidad para promocionar su actividad y estos nunca conllevan un desembolso de dinero. Según los datos recogidos, el método más utilizado con mucha diferencia han sido las redes sociales. Así, en un segundo plano quedan relegadas las revistas, la televisión, la radio y la prensa. Respecto a las redes sociales, con una actividad vinculada de manera directa y proporcional con las actividades de crowdfunding, actualmente la asociación cuenta con un significativo número de colaboradores, plasmados en la siguiente tabla: 
Tabla. 3. Número de seguidores de la Asociación por plataforma

\begin{tabular}{|cc|}
\hline Plataforma & As. Alba Pérez \\
Facebook & 129.875 seguidores \\
Twitter & 3.001 seguidores \\
teaming.net & 6.063 personas \\
migranodearena.org & $75.563 €$ \\
\hline
\end{tabular}

Fuente: elaboración propia (datos: marzo 2017)

En este punto, es necesario analizar con más detalle y resaltar el uso de la red social Facebook por parte de la Asociación, ya que la promoción de las donaciones a través de cualquiera de las plataformas expuestas anteriormente, se realiza principalmente a través de dicha red. Desde sus inicios, Facebook se ha erigido como la herramienta por excelencia utilizada por la entidad, canalizando de esta forma toda su información, peticiones y logros. El presidente de la asociación, padre de la niña que originó la creación de la organización, es el motor de todo cuanto sucede en la misma que ha sabido entender y potenciar las oportunidades brindadas por las nuevas tecnologías, en especial las redes sociales y las plataformas de crowdfunding. En este sentido, desde la entidad han establecido una estructura de donaciones fácilmente accesible a través de diferentes canales, dejando el espacio necesario para centrarse en la comunicación y difusión de las actividades y objetivos conseguidos al respecto. De esta forma y dando máxima prioridad a la transparencia, la asociación ha conseguido generar empatía por su objetivo en un número significativo de personas y fidelizar así su contribución.

\section{Conclusiones}

El presente trabajo demuestra cómo las tecnologías emergentes a través de nuevas herramientas, tales como las plataformas de crowdfunding, se están estableciendo como destacados instrumentos de innovación en la implicación de las personas en las organizaciones sociales. Cerrando el círculo, se hace imprescindible la necesidad e importancia de planificar, ejecutar y evaluar una buena estrategia de marketing y comunicación.

En este sentido y como reflejo de esta situación, destaca el caso analizado de la Asociación Alba Pérez, creado para la financiación de investigaciones sobre cáncer infantil. Es un caso singular tanto por la escala de los proyectos que maneja, como por la recurrencia participativa de mecenas en la misma. Tal y como señalan Sajardo y Pérez (2014), el importe medio de los proyectos financiados por crowdfunding varía en función de la plataforma de referencia. En este sentido, estos importes medios van desde los $4.000 €$ hasta los $500 €$. No obstante, la Asociación en cuestión ha conseguido finalizar con éxito proyectos de una escala 
muy superior, hasta $50.000 €$ en la plataforma migranodearena.org ${ }^{23}$, y crear grupos recurrentes de financiación de más de 7.000 miembros en la plataforma teaming.net ${ }^{24}$. Su presencia en los medios sociales se basa en su página de Facebook $^{25}$, que cuenta con más de 129.000 seguidores o su perfil de Twitter con más de 3.000 seguidores.

Gran parte del éxito de la iniciativa está en la capacidad de comunicación de su presidente Rafael, tanto en su intensidad como en los contenidos. Guiado por un objetivo claro, las tecnologías le han proporcionado las herramientas para hacerlo. No obstante, hay que destacar que en definitiva son solamente eso, herramientas. Sobre ellas, la organización ha sido capaz de crear una comunidad muy significativa que apoya y se compromete con la causa. Los datos estudiados han puesto de manifiesto la consistente tendencia ascendente y positiva que la organización está siguiendo. En este sentido, queda abierto el estudio de la sostenibilidad del modelo, ¿es la simplicidad y transparencia sinónimo de éxito? Además y a raíz del fuerte carácter personalista de la asociación, queda abierta a profundizar en el análisis una segunda pregunta: ¿es el proceso duplicable por otras entidades? Para poder responder con seguridad, cabría ampliar la muestra del caso de estudio a otras organizaciones y analizar su realidad.

Por último, desde este proyecto sí se puede concluir la existencia de la necesidad de profesionalización en el ámbito de la comunicación, sobre todo en la 2.0. Potenciar como característica principal de una entidad la transparencia es sin duda esencial, pero no suficiente para convertirse en una organización de referencia y lograr cumplir su misión. Todo lo demás, ya sean las plataformas o las redes sociales, son solo las herramientas que permiten a una entidad hacer partícipe a la sociedad de su labor profesional. Así, se ha demostrado que el marketing y la comunicación son una pieza fundamental del crowdfunding, siendo el vínculo existente incontestable y en consecuencia imprescindible para las organizaciones, trabajar por una estrategia sólida que amplíe las posibilidades de financiación

\section{Referencias bibliográficas}

Arroyo, I., Baladrón, A. y Martín, R. (2013) La comunicación en redes sociales: percepciones y usos de las ONG españolas. Cuadernos.info, $\mathrm{N}^{\circ} 32$, pp. 77-88.

Andreasen, A.R. y Kotler, P. (2003) Strategic Marketing for Nonprofit Organizations. 6th ed., Prentice-Hall, Upper Saddle River, NJ.

Batliwala S. (2002) Grassroots Movements as Transactional Actors. Implications for Global Society. Voluntas: International Journal of Voluntary and Nonprofit Organizations, Vol. 13, pp. 393-410.

Cejudo A. y Ramil, X. (2013) Crowdfunding. Financiación colectiva en clave de participación Ed. Asociación Española de Fundraising.

Chetty, S. (1996) The case study method for research in small-and medium-sized firms. International Small Business Journal, Vol. 15, № 1, pp. 73-85.

\footnotetext{
http://www.migranodearena.org/es/ong/723/asociacion-alba-perez-lucha-contra-el-cancer-infantil/ https://www.teaming.net/group/list?q=alba\%20perez

25 https://es-es.facebook.com/Fundaci\%C3\%B3n-ALBA-Perez-lucha-contra-el-c\%C3\%A1ncer-infantil$\underline{291319920916272 /}$
} 
Davies R. (2014) Civic Crowdfunding: Participatory Communities, Entrepreneurs and the Political Economy of Place. Massachusetts Institute of Technology.

Edgar, J. (2001) Don't cut spending on communications. The Chronicle of Philanthropy, Vol. 13, No 13 , pp. 43-44.

Fundación PwC (2014) Estudio sobre el presente y futuro del Tercer Sector Social en un entorno de crisis. En colaboración con la Fundación "la Caixa" y el Instituto de Innovación Social de ESADE.

García, G. y Ramírez, J.M. (2001) Imagen y comunicación en temas sociales. Libros Certeza, Zaragoza.

Herranz de la Casa, J.M. (2007) La gestión de la comunicación como elemento generador de transparencia en las organizaciones no lucrativas. CIRIEC-España, Revista de Economía Pública, Social y Cooperativa, № 57, pp. 5-31.

Kotler, P., Armstrong, G., Saunders, J. y Wong, V. (1999) Principles of Marketing. 2nd Edition. New Jersey. Prentice Hall.

Kotler, P. y Keller, K. L. (2006) Marketing Management. 12th Edition. Pearson, Prentice Hall. New Jersey.

Martín, R. (2010) La comunicación web del tercer sector: análisis de los sitios web de las ONGD calificadas por la AECID. Madrid: Universidad Rey Juan Carlos.

PETS (2012) Diagnóstico del Tercer Sector de Acción Social - II Plan Estratégico del Tercer Sector de Acción Social. Plataforma del Tercer Sector de Acción Social, Madrid.

Rialp, J. (2003) Escalas de Medición y Temas relacionados. Departamento de Economía de la Empresa, Facultad de Económicas, Universidad Autónoma de Barcelona.

Sampedro, V. (2006) Comunicación y tercer sector ¿Redes de nudos o vacíos? Nuevas tecnologías y tejido social. Documentación Social, No 140.

Santesmases, M. (2004) Marketing. Conceptos y estrategias. 5ta Edición. Madrid: Editorial Pirámide.

Sajardo, A. y Pérez, S. (2014) El sector No Lucrativo ante las nuevas herramientas de fundraising: ¿el crowdfunding antesala de la sociedad mecenas?, artículo aceptado para ser publicado a lo largo de este año en la Revista Vasca de Economía Social.

Vázquez, J.L. (2004) Pasado, presente y futuro de las dimensiones pública y social en el desarrollo conceptual del marketing. International Review on Public and Nonprofit Marketing, Vol. 1, No 1, pp. 9-34.

Villarreal, O y Landeta, J. (2010) El estudio de casos como metodología de investigación científica en dirección y economía de la empresa. Una aplicación a la internacionalización. Investigaciones Europeas de Dirección y Economía de la Empresa, Vol. 16, № 3, pp. 31-52.

Wolf, T. (1999) Managing a nonprofit organization in the twenty-first century. Simon \& Schuster Inc. USA.

Yin, R. (1989) Case Study Research. Design and Methods. Applied Social Research Methods Series, vol. 5, Sage Publications, London.

\section{Webgrafía}

Caldbeck, R. (2012) 2013 and The Evolution of Crowdfunding https://www.forbes.com/sites/ryancaldbeck/2012/12/26/2013-and-the-evolution-ofcrowdfunding/\#6209b4ed7d2e (último acceso 16 de marzo de 2017).

Kickstarter.com (2014) Creator handbook https://www.kickstarter.com/help/handbook/ (último acceso 16 de marzo de 2017). 
Lanzanos.com (2014) ¿Por qué la gente financia proyectos vía crowdfunding? http://www.slideshare.net/twomuchrs/conclusiones-por-qu-la-gente-financia-proyectosva-crowdfunding (último acceso 16 de marzo de 2017).

Startsomegood.com (2014) How it works http://startsomegood.com/HowItWorks (último acceso 16 de marzo de 2017).

Moisseyev, A. (2013) Effect of Social Media on Crowdfunding Project Results. University of Nebraska - $\quad$ Lincoln $\underline{\text { http://digitalcommons.unl.edu/cgi/viewcontent.cgi? } \text { article }=1043 \& \text { context=businessdiss }}$ (último acceso 16 de marzo de 2017).

Suárez, R. (2013) La Banca Electrónica En España. Máster Oficial en Banca y Finanzas, Universidades da Coruña. Facultad de Economía y Empresa. http://ruc.udc.es/dspace/bitstream/2183/11690/2/SuarezGomez_Roman_TFM_2013.pdf (último acceso 16 de marzo de 2017).

Suárez, F. (2012) Sistemas de Pago con móvil. Universidad Politécnica de Madrid - Escuela Universitaria de Ingeniería Técnica de Telecomunicación http://oa.upm.es/22580/1/PFC_FELIX_SUAREZ_CAMI\%C3\%910.pdf (último acceso 16 de marzo de 2017).

The crowdfunding Industry Report (2012) http://www.crowdfunding.nl/wpcontent/uploads/2012/05/92834651-Massolution-abridged-Crowd-Funding-Industry-

Report1.pdf (último acceso 16 de marzo de 2017).

The crowdatacenter (2014): Efunding and the state of the crowdfunding nation http://www.thecrowdfundingcenter.com/?page=report (último acceso 16 de marzo de 2017). 